\title{
Cuestionamientos a la enseñanza de la Economía desde una perspectiva compleja
}

\section{Violeta Glaría*}

\begin{abstract}
Resumen: Desde una perspectiva compleja aparecen claros cuestionamientos a los fundamentos y los razonamientos de la Economía Neoclásica (que es lo que se enseña en Chile cuando se enseña Economía), por ejemplo, la noción de emergencia: “el todo es más y menos que la suma de la partes” (Morin, 1981), la sociedad no es sólo un agregado de individuos. El conocimiento es incompleto: no hay información absoluta. Los contextos son determinantes para la comprensión de lo estudiado. Las esferas del conocimiento no están aisladas, surge la necesidad de la transdisciplinariedad. Desde el teorema de la incompletitud de Gödel las demostraciones matemáticas no aseguran la veracidad de los resultados ni la ausencia de subjetividad (además de haber comportamientos, como el humano, que no son reductibles a la lógica matemática). Es necesario replantearse las comprensiones de la Economía y su enseñanza. Diseñar nuevas cátedras de economía que incluyan otros contenidos, metodologías y reflexiones epistemológicas, ya que, dada la complejidad de los tiempos que afrontamos, y la importancia de las decisiones que se toman en nombre de la Economía, no podemos contar con profesionales que cuenten con una visión reduccionista de los fenómenos económicos.
\end{abstract}

Palabras Claves: complejidad, emergencia, economía y educación

\section{Questionings to the teaching of economics from a complex perspective}

\begin{abstract}
From a complex perspective the mains assumptions and the reasoning of Neoclassical Economy (that is what is being taught in Chile when Economics is taught) are questioned, for example, the concept of emergence: "Everything is more and less that the additions of the parts" (Morin, 1981), so societies are not just the sum of individuals. Knowledge is incomplete, there is not total information. Contexts are decisive to understand. The domains of knowledge are not isolated, emerge the need of transdisciplinarity. Since Gödel's theorem mathematical demonstration do not assure veracity of the results or the absence of subjectivity (besides there are complexities that are not reducible to mathematical knowledge, like human behavior). Is necessary to over think the comprehensions of traditional economics and to design new curses of economics that include other topics, methodologies and epistemological reflections, because, given the complexity of our times, and the important decisions that are being taking in the name of Economy, we can't have professionals that understand in a reductionist way the economical phenomena.
\end{abstract}

Key words: complexity, emergence, economy and education

*Universidad de Valparaíso, Valparaíso, Chile. Email: violetaglaria@socioeconomia-uv.cl 


\title{
Questionamentos ao ensino da Economia desde uma perspectiva complexa
}

\begin{abstract}
Resumo: Desde uma perspectiva complexa aparecem claros questionamentos aos fundamentos e os razonamientos da Economia Neoclásica (que é o que se ensina em Chile quando se ensina Economia), por exemplo, a noção de emergência: “o todo é mais e menos que a soma da partes” (Morin, 1981), a sociedade não é só um agregado de indivíduos. O conhecimento é incompleto: não há informação absoluta. Os contextos são determinantes para o entendimento do estudado. As esferas do conhecimento não estão isoladas, surge a necessidade da transdisciplinariedade. Desde o teorema da incompletitude de Gödel as demonstrações matemáticas não asseguram a a veracidade dos resultados nem a ausência de subjetividade (além de ter comportamentos, como o humano, que não são reductibles à lógica matemática). É necessário replantearse os entendimentos da Economia e seu ensino. Desenhar novas cátedras de economia que incluam outros conteúdos, metodologías e reflexões epistemológicas, já que, dada a complexidade dos tempos que enfrentamos, e a importância das decisões que se tomam em nome da Economia, não podemos contar com profissionais que contem com uma visão reduccionista dos fenómenos económicos.
\end{abstract}

Palavras-chave: complexidade, emergência, economia e educação

Recibido: 15.01.2010

Aceptado: 18.02.2010

$* * *$

\section{Introducción}

Antes de adentrarnos en la argumentación propia de este artículo resulta clarificador hacer una introducción acerca del quiebre paradigmático modernidad - posmodernidad, en el que se contextualiza la problemática a reflexionar.

El Paradigma Moderno, fruto del proyecto ilustrado, se yergue sobre la idea del "Progreso" como el horizonte que encamina los pasos de la humanidad y construye el nuevo "Hombre Universal”. La fe en Dios es reemplazada por la fe en la Razón, Descartes busca los principios claros y distintos de la realidad a través del análisis, las Ciencias Naturales se sustentan en los principios del Universalismos, el Naturalismo y la Objetividad (Hodgson, 1988) y se desarrollan rápidamente haciendo crecer la fe en las capacidades tecnológicas y el raciocinio. Estos elementos se conjugan en el Positivismo, que defiende el método científico como la forma de lograr conocimiento objetivo, busca leyes universales en el cosmos, razona linealmente y se sustenta en una concepción determinista del universo. Esta forma de concebir el mundo tuvo hondas consecuencias: se desarrolló la industrialización, los medios masivos de comunicación, la producción en masa. En términos económicos el progreso y la eficiencia se articularon con el paradigma clásico del liberalismo: libre mercado, expansión, beneficio y competencia. (Kurnitzky, 1994) 
Esta concepción moderna entró en crisis en el siglo XIX y XX: Todo lo sólido se desvaneció en el aire planteó Marx (1848), Einstening relativizó el tiempo a la velocidad de desplazamiento, Gödel demostró matemáticamente que la matématica no se explicaba a sí misma plenamente, Heisenberg planteó el principio de indeterminación y los físicos cuánticos no podían saber la velocidad de la partícula sí sabían la posición y no podía saber la posición sí sabían las velocidad. Desde la biología hubo quienes comprendieron la realidad como reflejo de la determinación estructural (Maturana y Varela, 1974), los físicos reconocieron el caos en el corazón de la materia, la Química, con Prigogine, encontró reacciones que parecían escaparse a la 2da ley de la termodinámica y las ciencias sociales generaron conocimiento.

Además, en términos históricos, la primera guerra mundial, la recesión del 29, la segunda guerra mundial, la descolonización de África y diversos conflictos humanos trizaron la fe en que la racionalidad, por sí sola, llevaría a la humanidad a conocer tiempos mejores. Tras la caída del muro de Berlín, el desmembramiento de la URSS y la aparente victoria incontestada del capitalismo neoliberal se agudiza este proceso de falta de sentido lo que profundiza la crisis de los “metarelatos" (Lyotard, 1979). Se quebró el sueño modernizador donde iban a triunfar los valores de la Ilustración y la Revolución Francesa, el mundo se mostró inestable y cruel, la moral no emancipó a la humanidad, la materia y el universo no se han mostrados caracterizables por los conceptos "claros y distintos" que buscaba Descartes. Surge la posmodernidad como una respuesta a este quiebre, por lo que se caracterizó por su incredulidad con respecto a los relatos y metarelatos, la deslegitimación del discurso especulativo y emancipatorio, y, como consecuencia, la crisis de la metafísica (Lyotard, 1979) . Se inaugura así, el pensamiento contemporáneo que augura el final del EstadoNación, el crepúsculo de la moral burguesa y la pérdida de seguridades ontológicas (Quezada, 2005)

La posmodernidad se yergue sobre el fracaso del proyecto moderno, es una perspectiva pesimista y crítica de la sociedad. Una de las opciones posmodernas entonces, es perderse en la deriva y a la superficialidad, no hay metarrelatos, todo es relativo, la emancipación humana ya no es accesible, por ende: todo puede dar lo mismo. El pensamiento es un divertimento, ya que la rigurosidad no es un seguro de objetividad. La realidad, y todas sus expresiones, son meros simulacros que pretenden articular un sentido, aunque vano, donde ya no existe (Baudrillard, 1974). La realidad se "desrealiza" al sumergirla en la incontrolable incertidumbre de los tiempos, y en las turbulencias de los fenómenos que se resisten a la reducción cartesiana, o a la elaboración de leyes generales para su comprensión.

Es en este contexto donde nace la teoría de la complejidad como un intento de articular conocimiento desde la ausencia de certezas. La posmodernidad rompe con la búsqueda del orden y el control propios del pensamiento moderno pero peligra con quedarse en el desierto de la 
incognición (Quezada, 2005). La complejidad, en cambio, pretende conocer desde la incertidumbre, desde la ausencia de leyes supremas. "Conocer y pensar no es llegar a una verdad absolutamente cierta, sino que es dialogar con la incertidumbre” (Morin, 1981:76).

La incertidumbre nos sumerge en un espacio multicausal de redes relacionales no lineales donde se conjuga lo nuevo con lo viejo, el orden con el desorden, lo coherente con lo contradictorio, y del cual emergen nuevos fenómenos, entre ellos, la autorganización. El estudio de estos fenómenos demanda la interrelación entre las esferas del saber, por ende, una postura cognitiva que no “mutile” el saber (Morin, 1981). Todo fenómeno ha de ser comprendido en su contexto, toda disciplina debe complementar su visión con otras disciplinas. La objetividad se vuelve subjetiva en la medida en que todo lo estudiado es estudiado por alguien desde una determinada perspectiva, epistemología, paradigma y condicionamiento cultural (Maturana y Varena, 1974). Con el desvanecimiento de la objetividad se desvanece la pretensión de llegar a La Verdad y todo conocer se vuelve relativo al sujeto y la sociedad que lo desarrolla. Todo conocimiento debe asumir humildemente que es sólo parcial, y mantenerse al borde de su propia destrucción. (Morin, 1981). Todo concepto, toda teoría es un acto de simplificación, que son útiles, indudablemente, pero que no deben dejar de ser considerados como eso: una simplificación. Lo real supera todo conocer humano, no hay definición ni sistema que abarque la totalidad de la realidad, no hay una Verdad Absoluta, hay saberes relativos. (Ibid)

En adelante el mundo será "rico en evoluciones impredecibles, lleno de formas complejas y flujos turbulentos, caracterizado por relaciones no lineales entre causas y efectos, y fracturado entre escalas múltiples de diferente magnitudes" (Fried, 2005:21). Se vuelve innecesaria la disyunción metodológica entre la deducción y la inducción, ambas son indispensables. Conocer se vuelve un movimiento pendular entre el todo y las partes, y entre las distintas perspectivas disciplinares.

Contextualizándose en este cambio paradigmático es que quiero incursionar en los cuestionamientos que, a mi juicio, se desprenden de la visión compleja en lo concerniente a la Economía Neoclásica y cómo esto debería tener serias repercusiones en los programas de las cátedras de economía. La Complejidad, como propuesta epistemológica, gatilla la necesidad de complejizar la comprensión económica y por ende replantear la enseñanza de esta disciplina las aulas.

\section{Economía Neoclásica y la Complejidad}

“Un estudio de la historia de la opinión es un preámbulo necesario para la emancipación de la mente”

John M. Keynes 
“La economía neoclásica elude el problema central que cuestiona el enfoque de la complejidad: imposibilidad de derivar el comportamiento del todo del comportamiento de las partes”.

\section{Rolando Astarita}

La Economía Neoclásica (que se articula bajo el paradigma moderno) se basa, por una parte, en el Individualismo Metodológico, y las corrientes que de él se desprenden como el Marginalismo. En estas corrientes de pensamiento lo central son las particularidades de las unidades básicas de comprensión, los individuos en forma aislada. No se considera que los individuos puedan tener objetivos que afecten el bienestar de otros individuos (Atilano y Sanchez, 2007) o que muchas de sus propiedades, como es, por ejemplo, tener poder, sean relacionales, de manera que la descripción exacta de un individuo exige la referencia a otros individuos (Covarrubias, 2002). Tampoco conciben que existan creencias y comportamientos supraindividuales que no son reductibles a creencias y comportamientos individuales, como los fenómenos culturales. De esta forma se omiten las emergencias de fenómenos nuevos a las particularidades de las partes como resultado de la interacción entre éstas.

“A partir de una concepción individualista se pretende construir un esquema de razonamiento que permita explicar el funcionamiento global o macro de las realidades sociales. Esto lleva a que se postulen algunas leyes generales de comportamiento del sistema generadas a partir de la agregación de las conductas individuales. ” (Coq, 2004:118)

Por otra parte la Economía Neoclásica, se basa en pensamiento filosófico llamado "Utilitarismo”, que se desarrolló en el siglo XVIII en el Reino Unido, según esta corriente de pensamiento lo esencial son los resultados y no los procesos. Los resultado son medidos de acuerdo a la Utilidad que generan, y ésta se utiliza como sinónimo de placer en el comportamiento de los individuos. La premisa es que las personas siempre van a querer maximizar el placer y minimizar el dolor, los que son cuantificables, y el egoísmo es la naturaleza esencial de los humanos. (Silva, 2003).

“La nueva ética (el cálculo utilitario), no siendo más que el estudio científico de las consecuencias del egoísmo racional, colocó al individuo en el centro. (...) Estas ideas proporcionaron un fundamento intelectual satisfactorio para los derechos de propiedad y la libertad del individuo para hacer lo que le plazca consigo mismo y con lo que le pertenece" (Keynes, 1926:63-64)

Los tres supuestos sicológicos sobre los que descansa el Utilitarismo son, según John Stuart Mill:

“Según el primero, el único objeto posible de voluntad o deseo es el placer o la ausencia de dolor. Con esto se afirma el egoísmo como único 
impulso sobre el cual hay que fundamentar la ética. Según el segundo supuesto, el placer es susceptible de medida o, lo que es lo mismo, todos los placeres son cualitativamente iguales. De aquí la posibilidad de una suma de placeres, que se calcularían según criterios de intensidad, duración, proximidad y seguridad. Y según el tercer supuesto, los placeres de distintas personas pueden compararse entre sí, ya que la sociedad es un agregado de individuos a los que deben darse las mismas oportunidades de placer." (Stuart, 1863:12)

De lo anterior se desprende que la conducta económica racional de los humanos es egoísta por naturaleza, que el placer es cuantificable, por lo que se puede trabajar con él matemáticamente, que los placeres son comparables entre sí y, (lo más importante desde el punto de vista de la complejidad), que una sociedad es una "agregado de individuos", de esta forma se niegan las emergencias propias de los fenómenos sociales. Según este planteamiento se puede explicar el todo por la sumatoria de las partes. Lo social surge como una simple agregación del comportamiento de las unidades básicas (Kugman, 1997). Sin embargo, desde la perspectiva de la complejidad, existen fenómenos que emergen de la interacción de las partes y que no son reductibles a la sumatoria de las mismas. "La organización de un todo produce cualidades o propiedades nuevas en relación con las partes consideradas de forma aislada: Las emergencias” (Morin, 1990:101)

Las elecciones racionales son las que maximizan la "felicidad" en un mundo de escasez. El rol de la economía es entonces asignar racionalmente (maximizando la felicidad) los recursos escasos de la sociedad. La preferencia individual, según este enfoque, es esencial para la elección racional. El concepto de racionalidad presupone una serie de elementos concurrentes en el proceso de decidir, se han de conocer "todas las alternativas posibles frente a un determinado estímulo, la existencia de una función de utilidad o preferencia que jerarquiza todos los conjuntos de consecuencias o el conocimiento perfecto de las consecuencias derivadas de cada una de las alternativas posibles a emprender" (March y Simon, 1987, en Coq, 2004:119).

Se parte de la premisa de la Información Absoluta (Conocimiento Perfecto) en donde se saben todas las alternativas posibles y sus consecuencias, conocimiento a partir del cual, según una lógica de costo beneficio, se puede discriminar racionalmente entre las distintas alternativas de la elección económica. Sin embargo, desde el punto de vista de la complejidad esto es imposible: "La conciencia de la complejidad nos hace comprender que no podremos escapar jamás a la incertidumbre y que jamás podremos tener un saber total: la totalidad es la no verdad.”(Morin, 1990:101)

Además, si analizamos el comportamiento económico desde un punto de vista más amplio nos damos cuenta de que: "No todas las elecciones de los hombre pretenden maximizar beneficios. No siempre elegimos racionalmente. No elegimos solos, sino grandemente influidos por los grupos a los que pertenecemos” (Etzioni, 1980:194) 
De acuerdo al Utilitarismo la Utilidad $(U)$ se define como una función del consumo de bienes del individuo (Varian, 1994), por lo que la máxima utilidad individual está relacionada con el máximo consumo de bienes.

$$
\begin{aligned}
& U=f(x) \\
& \mathrm{U}=\text { Utilidad } \\
& \mathrm{X}=\text { Bienes }
\end{aligned}
$$

Lo anterior conlleva el supuesto de que los Bienes representan la Utilidad que a su vez representa la Felicidad. Es decir, que a mayor consumo de bienes mayor Felicidad. Aunque cabe señalar que, de acuerdo a esta teoría, la felicidad nunca será completa porque las necesidades son infinitas. ${ }^{1}$

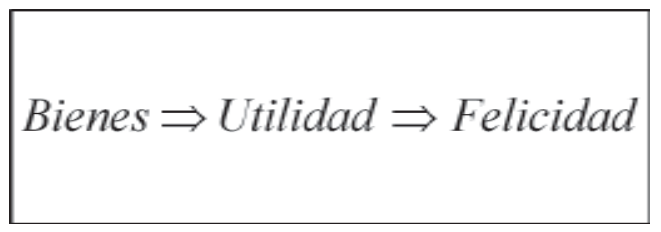

De que la Felicidad sea alcanzable a través del consumo de bienes y que estos se vean reflejados en la Utilidad aparece la necesidad de Maximizar esta función lo que está sujeto a la restricción presupuestaria de cada uno de los individuos.

$$
\begin{aligned}
& \text { Maximizar: } \\
& U=f(x)
\end{aligned}
$$

De esta restricción presupuestaria surgen las curvas de utilidad y su óptimo en la coincidencia con el ingreso disponible. Luego se desprende la curva de la demanda, la oferta, la noción de bienes complementarios, el excedente del consumidor, la elasticidad de la demanda, etc (lo esencial ya está expuesto en las líneas anteriores). La situación será óptima a niveles sociales en la medida que no exista ninguna forma de mejorar el bienestar de algunas personas sin empeorar el de otras, he aquí el Óptimo de Pareto. (Varian, 1994)

\footnotetext{
${ }^{1}$ Con esto se cimentan las bases teóricas del consumismo contemporáneo.
} 
En cuanto al comportamiento de las empresas se asumen los mismos supuestos que para el individuo, son intrínsecamente egoístas y su fin último es la maximización de las Utilidades que, en este caso, son sinónimo de beneficios monetarios (Ingresos - Costos).

Del supuesto de la deseabilidad de la maximización se llega a la necesidad del crecimiento económico constante (para aumentar los niveles de consumo, por ende de felicidad, y los beneficios de las individuos y empresas). Los economistas clásicos decían que se iba a poder crecer hasta el agotamiento de los recursos, existía la noción de límites. Los neoclásicos, por el contrario, hablaron del crecimiento infinito y lo justificaron con el reemplazo de los recursos agotados vía avances tecnológicos.

Los Neoclásicos tomaron, del pensamiento liberal de Adam Smith, la importancia de la libertad de emprendimiento y comercio, sin embargo, fue un comerciante quien acuñó el término laissez-faire y su popularización no estuvo a cargo de los economistas relevantes de la época.

"El lenguaje de los economistas se prestaba para el laissez- faire. Pero la popularidad de la doctrina debe dejarse a la puerta de los filósofos políticos de la época (...) La frase Laissez- faire no se encuentra en las obras de Adam Smith, Ricardo o Malthus. Ni siquiera la idea está presente en forma dogmática en alguno de estos autores. (...) La compaña política a favor del libre cambio, la influencia de la denominada escuela de Manchester, las declaraciones de autoridades económicas secundarias (...) fijaron el laissez - faire en la mente popular, como conclusión práctica de la economía política ortodoxa (...) la actitud cauta y nada dogmática de los mejores economistas no ha prevalecido contra la opinión general de que un laissezfaire individualista es lo que ellos debieron enseñar y lo que de hecho enseñaron” (Keynes, 1926:70)

De ahí se deduce otros de los supuestos utilizados, la indeseabilidad de la intervención estatal, y la importancia de dejar actuar la "Mano Invisible” del mercado para llegar a los puntos de equilibrio óptimos del sistema. (Smith, 1794)

A lo largo de la historia han sido muchos los críticos que han cuestionado una teoría que se yergue sobre premisas de dudosa representación de la realidad, ante estas crítica la reacción de los neoclásico ha sido plantear que todo modelo es una simplificación de la realidad, que lo que importan no es lo veraz de las premisas de partida sino el grado de explicación de la realidad al que se llega a través del modelo. Por ejemplo:

"Truly important and significant hypotheses will be found to have "assumptions" that are wildly inaccurate descriptive representations of reality, and, in general, the more significant the theory, the more unrealistic the assumptions. The reason is simple. A hypothesis is important if it "explains" much by little, that is, if it abstracts the common and crucial elements from the mass of complex and detailed circumstances surrounding 
the phenomena to be explained and permits valid predictions on the basis of them alone. To be important, therefore, a hypothesis must be descriptively false in its assumptions; it takes account of, and accounts for, none of the many other attendant circumstances, since its very success shows them to be irrelevant for the phenomena to be explained." (Friedman, The Methodology of Positive Economics en Hausman, 1994:14) ${ }^{2}$

Sin embargo, si se simplifica demasiado o si se asumen postulados totalmente alejados de la realidad se peligra con crear mundos paralelos. Todo modelo es una simplificación de la realidad, es verdad, pero no por eso va a poder cambiar axiomáticamente las características de la realidad.

“El problema reside en que cuando se hacen simplificaciones es necesario determinar en qué grado éstas se apartan de la realidad (...) si la distancia es demasiado grande se corre el riesgo de crear otra realidad (...) que no tiene punto de contacto con el mundo en que vivimos” (Astarita, 2008:5) ${ }^{3}$

Desde el punto de vista de la complejidad los fundamentos axiomáticos son vistos con desconfianza y no pueden establecerse acríticamente ni mantenerlos en el tiempo si hay indicios de que no dan cuenta en forma adecuada de la realidad. Si se establecen axiomas de partida, porque los modelos explicativos los requieren, estos deben ser constantemente revisados y se ha de mantener la conciencia de su existencia, y por ende, de sus limitaciones.

“¿Cuáles son mis fundamentos?, la ausencia de fundamentos, es decir, la conciencia de la destrucción de los fundamentos de la certidumbre. Esta destrucción de los fundamentos, propia de nuestro siglo, ha llegado al conocimiento científico mismo.(...) La complejidad no es un fundamento, es el principio regulador que no pierde nunca de vista la realidad del tejido fenoménico en la cual estamos y que constituye nuestro mundo (...) lo real es monstruoso. Es enorme, está fuera de toda norma, escapa, en última instancia, a nuestros conceptos reguladores”. (Morin, 1990:146).

\footnotetext{
2 "Encontrarán que las hipótesis realmente importantes y significantes tienen premisas que son representaciones descriptivas de la realidad muy poco apropiadas, y, por lo general, mientras más significativa la teoría, menos realistas son sus supuestos de partida. La razón es simple, una hipótesis es importante si explica “mucho" con "poco”, esto es, si abstrae los elementos cruciales y comunes de una masa de complejas y detalladas circunstancias que rodean el fenómeno a estudiar, y permite válidas conclusiones basándose sólo en dichas premisas. Para ser importante entonces, una hipótesis debe ser descriptivamente falsa en sus premisas y no tomar en cuenta otras circunstancias, ya que, su mismo éxito las muestra irrelevantes para el fenómeno a explicar.” (traducción propia)

3 "Por ejemplo uno de los supuestos axiomáticos del modelo de equilibrio general de Debreu es una sociedad en la cual todos los agentes son propietarios de todos los medios de producción. No se trata de una abstracción provisoria, porque jamás el modelo de Debreu levanta ese supuesto inicial. Aquí entonces no existen clases sociales; pero por eso mismo también ha desaparecido la sociedad capitalista. Esto es, la simplificación anuló lo que había que estudiar, la economía capitalista.” (Astarita, 2008:5)
} 
Sin duda, resulta tentador dejarse seducir por tan elegantes modelos matemáticos de curvas que se expanden y contraen y puntos de equilibrio precisos en los que la felicidad es máxima. "La belleza y simplicidad de una teoría semejante son tan grandes que es fácil olvidar que no se deduce de los hechos, sino de una hipótesis incompleta introducida en aras de la simplicidad.” (Keynes, 1926:77)

Por otra parte, la economía neoclásica, en su búsqueda de "cientificidad" ha tomado a las matemáticas como la única forma de validar su conocimiento, por ejemplo, Lipsey reconoce que para lograr ser validado en las revistas de economía de primera línea es necesario plantear modelos matemáticos aunque estos no aporten nada a la explicación del fenómeno analizado. (Lawson, 2005).

La elección de las matemáticas como forma de validar el conocimiento en la economía resulta una elección metodológica bastante cuestionable, ¿el comportamiento humano puede ser reducido a las lógicas matemáticas? ¿las subjetividades, los condicionamientos sociales, las conductas colectivas pueden ser expresados en términos matemáticos? Pareciera que no. Es cosa de estudiar el comportamiento de las crisis financieras recientes en las que el rol del pánico colectivo es esencial para comprender la magnitud de las crisis, su desarrollo y alcances. La economía no puede reducirse a la matemática porque los humanos no podemos pueden ser reducidos a la matemática. Cuando la economía se aleja de las reflexiones morales, éticas, sociales y políticas que le eran características y se reduce a modelos matemáticos se mutila la esencia de la comprensión económica.

"En el momento en que la economía abandona todo recurso a la intuición con el objeto de lograr una ciencia exacta, ajena a toda sustancia económica, entra en el mundo de los sistemas formales axiomáticos fundado en la nueva imagen del conocimiento matemático propuesto por Hilbert y seguido por Bourbaki; una imagen formal y en apariencia libre de determinaciones normativas pero que por su misma naturaleza abstracta se puede convertir en criterio de 'juicio objetivo' del campo de conocimiento económico. Esto sentencia el siguiente postulado: en cuanto quiere obtenerlo todo de sí misma, la teoría se hace inevitablemente ideológica.” (Franco de los Ríos, 2005:55)

La formalización matemática trae consigo otro problema: la lógica del formalismo Hilberiano. La complejidad nos enseña la coherencia de los contrarios, lo que Morin denomina el Principio Dialógico, que "permite asumir racionalmente la inseparabilidad de nociones contradictorias para concebir un mismo fenómeno complejo...” (Morin, 1990:101). En otras palabras, aquello que, desde la lógica formal, se entiende como contradictorio, pasa a ser complementario en un nivel de emergencia superior. Por ejemplo, el orden y el desorden pasan a ser elementos esenciales de la organización y conviven en el seno de la misma. (Morin, 1981) Esto, desde la perspectiva Hilberiana, sería completamente incongruente. 
Por otra parte, desde el teorema de la incompletitud de Gödel, las matemáticas dejaron de ser una lógica autocontenida que aseguraba la objetividad de los resultados. "El sistema formal, por tanto, ya no es prototipo de verdad absoluta, ya no hay verdades absolutas garantizadas lógicamente, la matemática ya no da tales garantías, la razón humana ya no puede asegurar con base en la matemática que se conozcan tal tipo de verdades, como lo pretendía el racionalismo.” (Nagel y Newman, en Campos 1994: 552)

En este sentido el aferro de la Economía Neoclásica a las matemáticas tienen aún menos sentido, ya que si mediante ellas no se puede asegurar la objetividad, léase cientificidad, de los resultados, cabría preguntarse ¿qué beneficio conlleva esta elección metodológica que, como hemos visto, implica una simplificación que atenta en contra de alguno de los elementos básicos del fenómenos estudiado?

Otro de los elementos esenciales de la perspectiva compleja es la importancia de la comprensión de los contextos en los cuales se desarrolla el fenómeno estudiado, el movimiento pendular entre todo y las partes, el que hablé al comienzo. Ante esto la economía Neoclásica es totalmente ciega, no incorpora los contextos culturales, políticos, espaciales, territoriales ni ecosistémicas dentro de su análisis.

Los contextos culturales determinarán los comportamientos económicos, individuales y colectivos, no existen comportamientos económicos ajenos a las características culturales que se les dan sentido. La Economía, en las distintas sociedades, se encuentra inmersa en las relaciones sociales de dicho grupo humano. (Guerra. A. Pablo, 1999)

Las condiciones políticas son el marco institucional que determina las posibilidades de los agentes económicos. El Estado con sus instituciones, regulaciones y estructuras (o ausencia de éstas) condicionan el devenir de las decisiones económicas. Esta situación se extienden nivel supranacional, las instituciones supranacionales influyen y condicionan, a su vez, las políticas nacionales.

El territorio, elemento esencial del contexto, tampoco se encuentra presente en el análisis económico convencional.

"El razonamiento económico imperante (sustentado sobre una base metodológicamente individualista derivada de una concepción positivista de la ciencia) se basa en la explicación del proceso de generación de costes y precios a partir de la concurrencia de dos únicos factores productivos: capital y trabajo. Estos factores se suponen homogeneizados (...) En este contexto, la introducción de la variable espacial supone una complejización considerable de este esquema. En su acepción más simple, la introducción el espacio en el análisis supone la existencia de un nuevo tipo de coste, el transporte, que dificulta o incluso imposibilita la obtención de una situación de equilibrio estable. En sus acepciones más complejas la introducción de la variable espacial (que pasa a ser denominada territorio) supone 
el reconocimiento de que los factores productivos no son homogéneos. No lo es el trabajo, que se ve afectado por una serie de relaciones sociales, culturales e institucionales específicas. Pero tampoco lo es el capital que, como relación social que es, se ve influido por los mismos elementos. Por tanto, en su forma más compleja la inclusión de la variable territorial implica una reconsideración de toda la conceptualización teórica realizada de los fenómenos económicos” (Coq, 2004:121).

Los contextos ecosistémicos, por otra parte, imponen límites a las actividades humanas. Las sociedades humanas y sus sistemas económicos son, a fin de cuentas, sistemas biológicos, y todo sistema biológico tiene límites delicados sobre los cuales su desarrollo se vuelve insustentable. (Orrego, 1998).

Y, finalmente, otro de los problemas de la Economía Neoclásica desde esta visión compleja, es que no busca complementar su visión con otras disciplinas. Es un conocer "Autista"4 que no admite dialogar con los otros saberes para que complementen su visión. De este modo la comprensión del fenómeno económico se ve mutilada de su complejidad, dejando apenas un cúmulo de modelos matemáticos y suposiciones que pretenden explicar lo que, en realidad, se niegan a estudiar.

Resumiendo, las principales críticas a la Economía Neoclásica desde una perspectiva compleja son: que niega la posibilidad de hayan fenómenos emergentes en los grupos humanos, que parte de supuestos altamente cuestionables y se aferra a ellos en forma acrítica y definitiva, que asume la objetividad en términos epistemológicos, que busca validar su cientificidad utilizando demostraciones matemáticas cuando éstas no aseguran dichos resultados (Gödel), que pretende poder reducir las complejidades sociales a la lógica matemática, que no incorpora la coherencia de los contrarios, el Principio Dialógico, dado el formalismo Hilberiano que sustenta su búsqueda de las demostraciones matemáticas, que asume que existe la posibilidad de la Información Absoluta (elemento esencial de la Racionalidad Económica), siendo que esto resulta un imposible dado el carácter de parcial de todo conocimiento., que descontextualiza los procesos económicos, omitiendo los contextos culturales, políticos, territoriales y ecosistémicos, y, finalmente, que a pesar de todas sus miopías, no busca complementar su conocimiento con el de otras disciplinas que le ayudarían a comprender aquellos elementos que le resultan invisibles dado su propio cuerpo teórico.

\section{A modo de conclusión}

La Economía Neoclásica es insuficiente (y puede llegar a ser perjudicial si es lo único que se enseña) para la comprensión de las realidades

\footnotetext{
${ }^{4} \mathrm{Al}$ respecto cabe resaltar la "Carta Abierta de Estudiantes de Economía del Mundo" que se denominan "Post Autistas", en alusión a la superación del autismo propio de la Economía Neoclásica.
} 
económicas actuales. Los humanos generamos emergencias que influencian nuestro comportamiento económico y que, a la vez, son influencias por éste. Las decisiones económicas no sólo son técnicas, son políticas, culturales y ecosistémicas, y en dichos términos merecen ser reflexionadas.

Cabe preguntarse ¿cuáles son las emergencias que generamos con nuestras sociedades cuyos comportamientos económicos parten de las premisas del egoísmo intrínseco, la maximización del consumo como sinónimo de la felicidad y sin otros límites que la restricción presupuestaria, necesidades infinitas, la maximización de los beneficios, el crecimiento infinito, el laissez-faire, la pretensión de una racionalidad económica que se sustenta en una información absoluta? Quizás algunas de esas emergencias puedan ser: ¿el deterioro ecosistémico creciente, la pobreza de más de la mitad de la humanidad, la progresiva concentración de la riqueza, la proliferación impune de negocios muy lucrativos pero dañinos para el bienestar común como el de las drogas o las armas?

El estudio de la economía no puede pretender que ésta funciona en un espacio abstracto en donde las consecuencias sociales y ecológicas de su comportamiento puedan ser simplemente invisibilizadas. La economía se desarrolla en un espacio particular, con límites claros y frágiles y con sujetos sociales que se ven afectados por cada una de las decisiones que se toman en su nombre. Todo sistema social, por muy antropocéntricamente que se estructure es un sistema biológico. (Orrego, 1998) y como tal no puede crecer indefinidamente. Hace casi dos décadas que Herman Daly (1992) planteó que no se puede crecer infinitamente en un mundo finito, una afirmación que podría parecer una obviedad pero que no ha sido escuchado por el Main Stream de la Economía. Es por esto que Nicholas Georgescu-Roegen ${ }^{5}$ se pregunta ¿qué le enseña la biología a la economía? Y la respuesta es: ¡i¡Límites!!!

Los límites biológicos no pueden ser sobrepasados por un sistema económico que se fundamenta en un paradigma de crecimiento infinito sin llegar al colapso del sistema. Éste es uno de los ejemplos en el que un análisis particularizado en una sola área del saber puede tener peligrosas consecuencias para el desarrollo de la humanidad en su conjunto.

Todo esto debe ser reflexionado, discutido y planteado en las aulas de clases donde se enseña economía, son demasiadas las decisiones que se toman en nombre de la economía para que, quienes saben de esta materia, sigan imaginando el mundo con comprensiones decimonónicas, reduccionistas, lineales y deterministas. El desafío del presente es fundamentar rigurosidad en el pensar aunque no haya certezas absolutas. La magnitud de los problemas actuales demanda un enfoque transdisciplinario de los mismos. Las escalas globalizadas de los fenómenos involucran tal

\footnotetext{
${ }^{5}$ Federico Aguilera Klink y Vicent Aleáutara (1994), De la economía ecológica a la economía ambiental. Ediciones FUHEM, Madrid.
} 
complejidad que el pensamiento parcelado no puede más que llevar a la incomprensión de los problemas estudiados. (Max-Neef, Elizalde y Hopenhayn, 2001) Una nueva metodología, que sepa trascender las barreras disciplinarias manteniendo la rigurosidad debe implementarse en las investigaciones futuras, y sobre todo, en la toma de decisiones y planes de desarrollo. “...tenemos que comprender que nuestra lucidez depende de la complejidad del modo de organización de nuestras ideas...” (Morin, 1990:101)

Para lograr este objetivo la educación es la herramienta esencial. Los nuevos profesionales que se inserten al "mercado laboral” (reduccionista expresión para denotar el tejido social de nuestro país) deben tener otras herramientas de reflexión y comprensión de los fenómenos y problemáticas que plantea el siglo XXI, calentamiento global, sobrepoblación, migraciones, pérdida de resiliencia ecosistémica, pérdida de biodiversidad, contaminación, crisis financieras, guerras, nuevas alianzas estratégicas, cambios paradigmáticos, avances tecnológicos constantes, entre otros.

"Un abordaje que haga honor a la complejidad debe ser capaz de conjugar de múltiples maneras los distintos niveles del cambio, explorar sus articulaciones, construir itinerarios según las problemáticas particulares que se presenten en cada indagación específica.” (Najmanovich, 2002, citado en Carrizo, Espina y Klein, 2003:8).

Puede que la misma supervivencia de nuestra especie esté en juego ya que estamos llevando a los sistemas biológicos de los que somos parte a los límites de su sustentabilidad. Las nuevas generaciones deben contar con nuevas herramientas epistemológicas y cognitivas con que afrontar estos desafíos y el paradigma de la complejidad es una de esas herramientas.

Y siendo la educación la principal responsable de formar a estas nuevas generaciones con las competencias y comprensiones necesarias para enfrentar nuestra época y las venideras, recae sobre ésta una gran responsabilidad. Parafraseando a Einstein: no podemos pretender que las cosas cambien si seguimos haciendo lo mismo y, de seguro, no podemos pretender que dejemos de hacer lo mismo si seguimos pensando igual, e, indudablemente, no podremos cambiar el modo de pensar sin cambiar el modo de educar. 


\section{Bibliografía}

Astarita, Rolando (2008), Nota de clase para Desarrollo Económico, Facultad de Ciencias Económicas, Universidad de Buenos Aires, Buenos Aires. http://www.iade.org.ar/modules/noticias/article.php? storyid $=2275$

Atinao, José y Sánchez, José Manuel (2007), “Fundamentos morales de la economía; una relectura del problema de Adam Smith”, Revista de Economía Institucional, vol. 9, № 16, primer semestre/2007, pp. 63-87, Coriña.

Baudrillard, Jean (1974), La sociedad de consumo. Sus mitos, sus estructuras, Editorial: Plaza y Janés, Barcelona.

Campos, Alberto (1994), Axiomática y geometría: desde Euclides hasta Hilbert y Bourbaki, Universidad Nacional de Colombia, Editorial Unibiblos, Bogotá.

Carrizo, Espina y Klein (2003), Transdisciplinariedad y Complejidad en el Análisis Social, Gestión de las Transformaciones Sociales (MOST), Ediciones UNESCO, Organización de las Naciones Unidas para la Educación, la Ciencia y la Cultura.

Coq, Daniel (2004), “Epistemología, Economía y Espacio/Territorio: del individualismo al holismo”, Revista de Estudios Regionales, enero - abril, n. 69, Universidad de Andalucía, Málaga.

Covarrubias, Isaías (2002), “Enfoque Sistémico e Individualismo Metodológico: una aproximación”, Revista Contribuciones a la Economía, ISSN 16968360. http://www.eumed.net/cursecon/colaboraciones/ ICM-ESIM.htm

Daly, Herman (1992), "From adjusment to sustainable development: the obstacle of free trade” en Loyola of Los Angeles Int. Comp. Law J., N ${ }^{\circ} 15$

Etzioni, Amitai (1980), La sociedad activa. Una teoría de los procesos societales y políticos, Aguilar, Madrid.

Franco de los Rios, Camilo Andrés (2005), “El Formalismo Axiomático en Economía”, Cuadernos de Economía, V.XXIV, n. 43, Bogotá, pág. 35 63.

Fried, Schnitman, Dora (2005), Nuevos paradigmas, cultura y subjetividad, Editorial Paidós, Buenos Aires.

Guerra, Pablo (1999), “Análisis socioeconómico solidario de las economías alternativas”, Revista Iberoamericana de Autogestión y Acción Comunal, No.34, Madrid. http://www.riless.org/biblioteca_desarrollo. shtmlcmd[223]=x22381ad81225dfc8b14d26911c1fd96d893 
Hausman, Daniel M. (1994), The Philosophy of Economics, an Antology, Segunda Edición, Press Sindicate of the University of Cambridge, Cambridge.

Hodgson, G. M. (1995), Economía y evolución: Revitalizando la Economía, Colegio de Economistas, Madrid.

Keynes, John M. (1985), Ensayos sobre intervención y liberalismo, Biblioteca económica, Ediciones Orbi. (original “The end of laissez-faire”, 1926).

Kurnitsky, Horst. (1994), “¿Qué quiere decir modernidad?”, La Jornada Semanal, No. 222, México.

Klink, Federico y Aleáutara, Vicent, (cáp. 3 Nicholas Georgescu-Roegen) (1994), De la economía ecológica a la economía ambiental, Ediciones FUHEM, Madrid.

Kruman, P (1997), La Organización espontánea de la Economía, Editorial Bosch, Barcelona.

Lawson, T. (2005), “The nature of heterodox economics”, Cambridge Journal of Economics, Cambridge. www.econ.cam.ac.uk/faculty/lawson.

Lyotard, Jean-Francois (1990), La condición posmoderna. Informe sobre el saber, Editorial Cátedra, Madrid.

Maturana, Humberto y Varela, Francisco (1974), El árbol del conocimiento, Editorial Universitaria, Santiago.

Marx, Karl y Engels, Frederick (1948), Manifiesto Comunista, Editorial Alambra, Longman 1995.

Max-Neef, Manfred; Elizalde, Antonio y Hopenhayn, Martín (2001), Desarrollo a Escala Humana, Segunda edición, Nordan Comunidad, Montevideo.

Morin, Edgar (1981), El Método Tomo I. La naturaleza de la naturaleza, Editorial Cátedra, Madrid.

Idem (1990), Introducción al Pensamiento Complejo, Gedisa Editorial, Madrid.

Orrego, Juan Pablo (1998), La tragedia del Bosque chileno, Defensores del Bosque Chileno, Santiago.

Quezada, Freddy (2005), “El pensamiento contemporáneo”. CIELAC, Centro Interuniversitario de Estudios Latinoamericanos y Caribeños, Universidad Politécnica, Managua. http://bibliotecavirtual.clacso.org.ar/ar/libros/ cielac/quezada.doc 
Silva Vegas, Gerardo (2003), “Comentarios sobre el paradigma Neoclásico: Epistemología de la Economía”, Revista Académica Estudios de Ciencias Administrativas de la Unidad de postgrado de la Facultad de Ciencias Administrativas de la Universidad Nacional Mayor de San Marcos, Año I, Volumen I.

Smith, Adam (1794, original), La riqueza de la Naciones, Ediciones Orbis S.A., Buenos Aires 1983.

Stuart Mill, John (1863, original), El Utilitarismo, Ediciones Aguilar, Buenos Aires 1974.

Varian, Hal (1994), Microeconomía Intermedia, un enfoque moderno, Editor Antoni Bosch, Barcelona. 